\title{
Segregation Processes in Segmented Polyurethaneurea Solutions Observed by Fluorescence Depolarization
}

\author{
Lameck Banda, Qui TranCong-Miyata, ${ }^{\dagger}$ and Shunji Nomura \\ Department of Polymer Science and Engineering, \\ Kyoto Institute of Technology, Matsugasaki, Kyoto 606-8585, Japan
}

(Received March 18, 1999)

\begin{abstract}
The onset of a segregation process in segmented polyurethaneurea (SPUU) solutions was studied by fluorescence spectroscopy using free fluorescein as a probe. For SPUUs with a given molecular weight and a constant ratio of the hard to soft segments, the time-resolved fluorescence measurements reveal the environmental inhomogeneity on the excited lifetime of fluorescein. It is shown in this work that the critical concentration for the segregation process detected by fluorescence spectroscopy through the transition of the anisotropy ratio of the fluorescein occurs at a concentration lower than that observed by small angle X-ray scattering (SAXS). These experimental results indicate that fluorescence depolarization can be used as an alternative method to investigate the structural transition in SPUU solutions.

KEY WORDS Polyurethaneurea/Lifetime / Depolarization / Microdomain /
\end{abstract}

Segmented polyurethaneurea (SPUU), are thermoplastic block copolymers which have mechanical properties similar to those of conventional elastomers. They belong to a class of (A-B) type multi-block copolymers composed of two chemically dissimilar chain segments called hard and soft segments. ${ }^{1}$ The hard segments act as multifunctional junctions, playing a role of both physical crosslinks and reinforcing fillers. These segments are formed by reacting an aromatic diisocyanate with a low molecular weight diol and/or diamine. On the other hand, the soft segments impart the elastomeric properties which are usually the aliphatic polyethers or polyester macroglycols. At low temperature, the low melting soft segments are incompatible with the hard segments of high melting point, leading to a microphase separation.

Similar to multiphase polymeric materials, it is expected that the physical properties of SPUUs critically depend on the phase separation between the hard and soft segment components. In the past decade, a large number of studies were carried out to establish the correlation between the morphology and the mechanical as well as the thermal properties of polyurethanes. ${ }^{2,3}$ Therefore, understanding of microphase separation processes is important for the control of the physical properties of these materials. Microphase separation of SPUUs has been studied by a number of experimental techniques including small angle X-ray scattering (SAXS). ${ }^{4}$ However, SAXS requires a certain threshold for the contrast in electron density difference to be observable and therefore, it would be interesting to observe these segregation processes by some other alternative methods which are sensitive to the structural transition in solutions of SPUU. Fluorescence depolarization is a promising candidate because transitions in the local structures can be detected through the changes in transport coefficients such as rotational relaxation time. This provides an alternative method to observe the microphase separation from the dynamic point of view. Furthermore, the rotational relaxation time is directly

\footnotetext{
† To whom correspondence should be addressed.
}

related to the so-called local viscosity which is an indicator of the local structures of the polymers. Thus, a change in local structures at the segmental level can be detected by measuring the anisotropy ratios of the polymer segments or of a fluorescent probe dispersed in the solution.

In this paper we experimentally show that fluorescence depolarization is a sensitive and useful technique to follow the segregation behavior of these multiphase polymer solutions. First, the experimental procedure is described in detail. Subsequently, the photophysical properties of the molecular probe fluorescein are investigated in SPUU solutions of various concentrations. The steady-state anisotropy and excited lifetimes of fluorescein in SPUU solutions are determined. From the dependence of the anisotropy ratio on the concentration of the polymers, information on the segregation process in SPUU solutions is extracted. This structural transition is finally discussed by comparing with the experimental data obtained from SAXS.

\section{EXPERIMENTAL}

\section{Samples}

Polyurethaneurea (SPUU) used in this work was provided by Asahi Kasei Co. $\left(M_{n}=55000, \alpha=1.8\right)$, where $\alpha$ is the molar ratio of the hard to the soft segments. The polymer was purified by reprecipitation using $N, N$ dimethylacetamide (DMAc) as good solvent and methanol as non-solvent. Subsequently, it was dried for $24 \mathrm{~h}$ in vacuo prior to the experiments. Solutions were prepared by using anhydrous DMAc of spectroscopic grade (Wako Chemicals, Japan). Free fluorescein (Fl, Wako) was used as the probe without further purification. The concentration of $\mathrm{Fl}$ in all the solutions was kept at $3.4 \times 10^{-5} \mathrm{M}$ to avoid the depolarization caused by concentration quenching which becomes significant at the concentration higher than $10^{-4} \mathrm{M} .^{5}$ The $\mathrm{pH}$ for all the solutions was 7 . In order to minimize the spatial inhomogeneity of samples, only solutions which can be prepared by mechanically stirring were used. All the experiments were performed at $26^{\circ} \mathrm{C}$ using non-degassed 
<smiles></smiles>

Soft Segment (MDI-PTMG)<smiles></smiles>

Hard Segment (MDI-EDA)<smiles>O=C(O)c1ccccc1-c1c2ccc(=O)cc-2oc2cc(O)ccc12</smiles>

Fluorescein

Figure 1. Chemical structures of the polyurethaneurea and the fluorescent probe used in this study.

Table I. Chemical composition of the SPUU

\begin{tabular}{cc}
\hline$\alpha$ Value & PTMG/MDI/EDA + DEA \\
\hline 1.8 & $1.0 / 1.8 / 0.80$ \\
\hline
\end{tabular}

solutions. The characteristics and the chemical structures of polyurethaneurea and $\mathrm{Fl}$ used in this work are shown in Figure 1 and Table I, respectively.

\section{Absorption Measurements}

Absorption spectra of Fl in DMAc solutions of SPUU were measured by using a UV-Vis spectrophotometer (Shimadzu, Model UV-160).

\section{Refractive Index Measurements}

The change in polarity of solutions with polymer concentration was obtained from the measurement of their refractive index $(n)$ using a refractometer (Atago, Model 2T, Japan). All the experiments were performed at $26^{\circ} \mathrm{C}$ using the D-line $(589 \mathrm{~nm})$ of a sodium lamp. The dielectric constant $(\varepsilon)$ of solutions with different concentrations of SPUU was then calculated by using the relationship;

$$
\varepsilon=n^{2}
$$

\section{Fluorescence Measurements}

The stationary-state anisotropy were obtained from the two polarized components of fluorescence intensity measured on a fluorometer (Shimadzu, Model RF$5300 \mathrm{PC}$ ) using quartz cuvettes with $1 \mathrm{~cm}$ path length. Fl was excited at $492 \mathrm{~nm}$ by using a Xe lamp and its fluorescence was detected at $544 \mathrm{~nm}$. Details of the steady-state and time-resolved measurements conducted are as outlined in ref 5,6, and 7 .

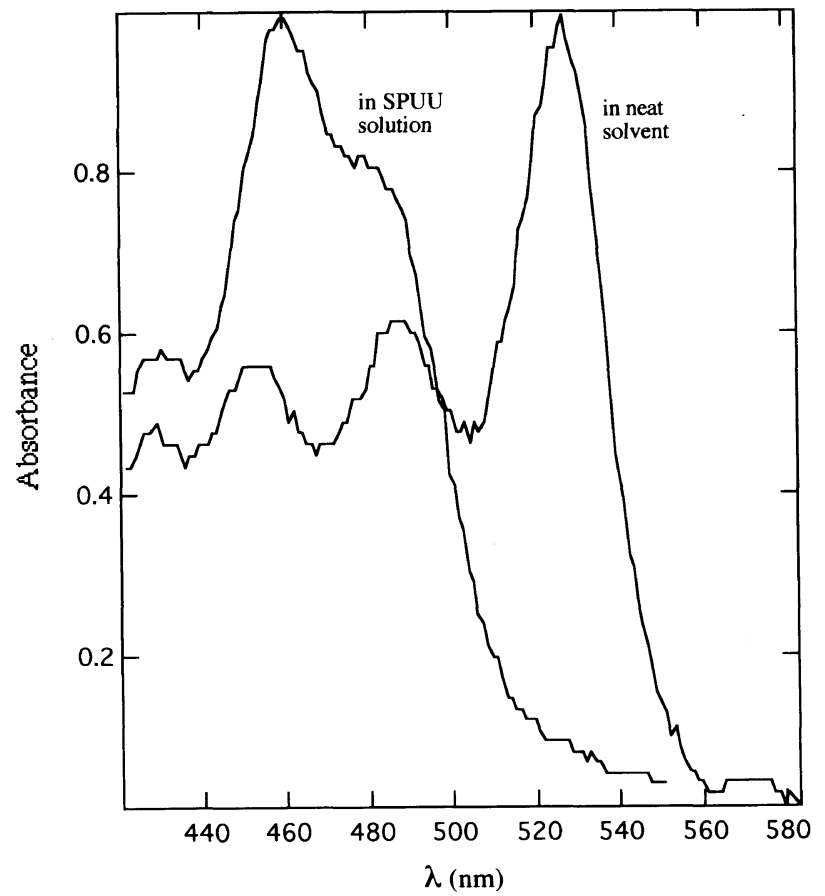

Figure 2. Normalized absorption spectra of $F 1$ in neat solvent (DMAc) and in a SPUU solution. The dimer peaks which are significant in neat solvent is weakened in the polymer solution.

\section{SAXS Measurements}

The onset of segregation in these solutions was observed by SAXS. The SAXS measurements were carried out at the Synchrotron Facilities of the National Laboratory for High Energy Physics (Tsukuba, BL-10C line). Briefly, the incident X-ray beam from synchrotron orbital radiation (SOR) with a wavelength of $1.488 \AA$ selected from a $2.5 \mathrm{GeV}$ storage ring was point focused on the sample using a double-flat monochromator combined with a cylindrical mirror. The sample thickness was about $1 \mathrm{~mm}$ and was sealed in a stainless steel cell with thin Mylar windows (poly(ethylene terephthalate) (PET), Teijin) each with a thickness of about $5 \mu \mathrm{m}$. Detection of the scattered X-ray was done by a onedimensional position sensitive proportional counter (PSPC, 512 channels), placed at a distance of $1.9 \mathrm{~m}$ from the sample holder. Sampling time was maintained at $5 \mathrm{~min}$. Data analysis was preceded by correcting the scattering intensity by subtracting the absorption of the sample and the two Mylar windows from the total intensity. The experimental temperature was controlled with a precision of $\pm 0.5^{\circ} \mathrm{C}$ and all the curve fitting was carried out using a nonlinear least squares program with regression.

\section{RESULTS AND DISCUSSION}

\section{Photophysical Properties of Fluorescein in SPUU Solu- tions}

Since the absorption spectra of the neat SPUU in the long wavelength side disappear above $460 \mathrm{~nm}$, the intrinsic fluorescence of the polymer is negligible under excitation with $492 \mathrm{~nm}$. Figure 2 shows the absorption spectra of Fl in neat DMAc and in DMAc solutions of SPUU. In both the neat solvent and in the polymer solutions, there exist two new peaks on both sides of the 


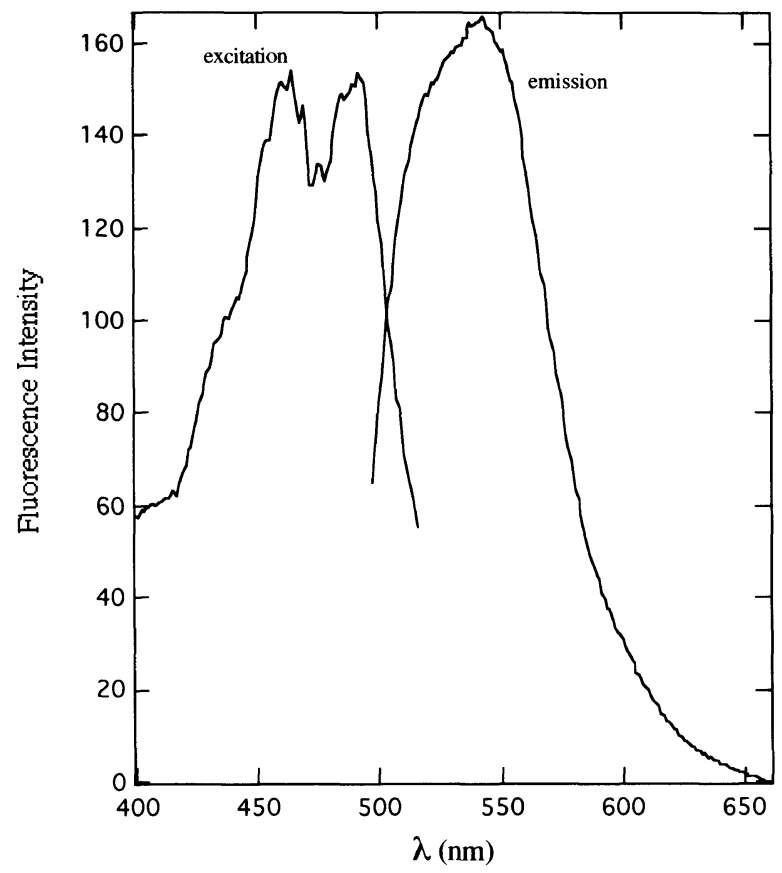

Figure 3. Excitation and emission spectra of $\mathrm{Fl}$ in a $20 \mathrm{wt} \% \mathrm{DMAc}$ solution of SPUU.

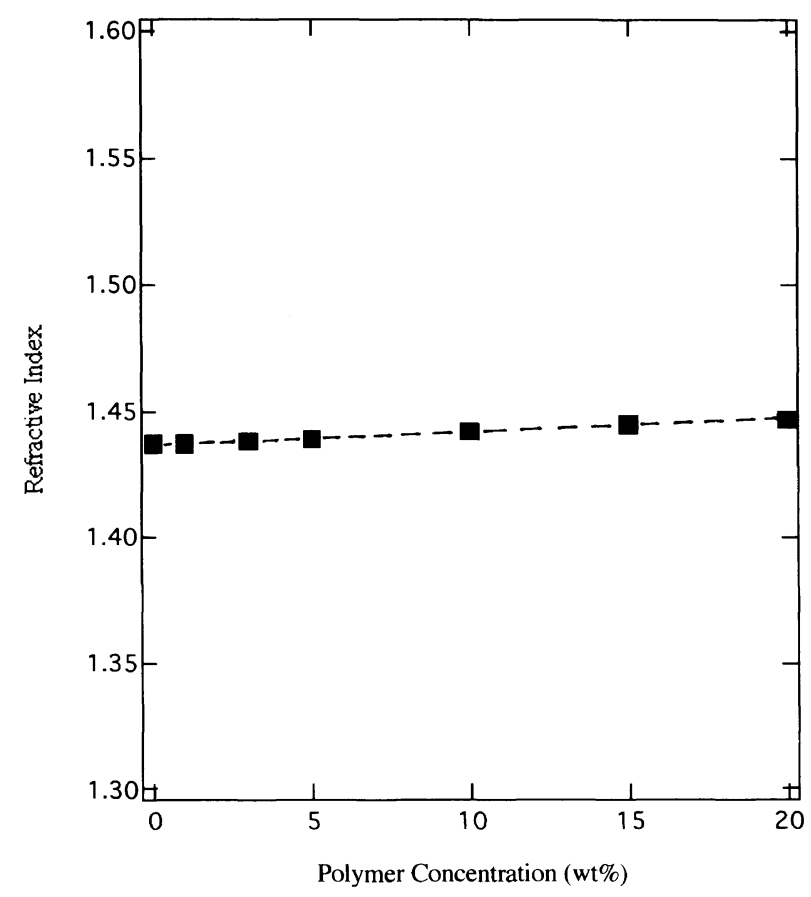

Figure 4. Dependence of the refractive indices of the SPUU polymer solutions on polymer concentration.

monomer peak of $\mathrm{Fl}$ at $490 \mathrm{~nm}$. These additional peaks have been identified as the absorption spectra of the dimers of types I (overlapping dimer) and II (side-by-side dimer) ${ }^{8}$ On the other hand, the absorption peak of the dimer type II on the long wavelength side almost disappears in the presence of SPUU. Since it is wellknown that urea weakens the hydrogen bondings, ${ }^{9,10}$ the presence of the urea moieties on the SPUU molecules might be responsible for the change in the absorption spectra of $\mathrm{Fl}$ in the solution. The fluorescence spectra of $\mathrm{Fl}$ in a $20 \mathrm{wt} \%$ of SPUU solution are shown in Figure 3 where the typical emission band of $\mathrm{Fl}$ monomer can be seen at $544 \mathrm{~nm}$ which remains almost unchanged with the polymer concentration upto $25 \mathrm{wt} \%$. Shown in the same figure are the corresponding excitation spectra monitored at $544 \mathrm{~nm}$ where the absorption spectrum of $\mathrm{Fl}$ in Figure 2 is recovered. On the other hand, as illustrated in Figure 4, the change in the refractive-index of DMAc solutions of polyurethaneurea is less than $1 \%$ upto $20 \mathrm{wt} \%$ of the polymer. It is worth noting that the emission peak of $\mathrm{Fl}$ only shifts $c a .1 \mathrm{~nm}$ toward the shorter wavelength side. Together with this insensitivity of the fluorescence spectra against the SPUU concentration, the result obtained in Figure 4 suggests that the increment of solution polarity with the polymer concentration does not seem to have a significant effect on the photophysical processes of $\mathrm{Fl}$.

In order to gain further information on the photophysical processes of Fl in DMAc solutions of SPUU, lifetime measurements were carried out under various experimental conditions.

Fluorescence Lifetime of Fluorescein in SPUU Solutions

Figures 5(a)-(c) show respectively the fluorescence decay curves of $\mathrm{Fl}$ in neat solvent, $20 \mathrm{wt} \%$ dimethylacetamide solution of SPUU and in a polytetramethylene glycol solution, the soft segment of SPUU. It was found that, in neat solvent, the decay curve is a single exponential function of time with an excited lifetime of ca. $3.8 \mathrm{~ns}$. However, in the presence of SPUU, the fluorescence decay curve becomes non-exponential and is well fitted to a sum of two exponential functions of time as illustrated in Figure 5(b):

$$
I(t)=F_{1} \exp \left(-\frac{t}{\tau_{1}}\right)+F_{2} \exp \left(-\frac{t}{\tau_{2}}\right)
$$

where $\tau_{1}$ and $\tau_{2}$ are the excited lifetimes of the short- and long-lived components, and $F_{1}$ and $F_{2}$ are their corresponding fractions. The best fit parameters for the decay curves are provided in Table II.

This multiplicity of Fl lifetimes was also observed in the neat soft segment, i.e., poly(tetramethylene glycol) (PTMG), measured at the same temperature. From the fact that:

(1) The solubility of Fl in solvent DMAC is better than in the polymer,

(2) The absorption spectra of Fl in the 3 environments: neat DMAc, SPUU solution and neat PTMG exhibit the characteristic absorption of dimers,

(3) Dimer of Fl does not fluoresce 8,11 and the fluorescence of monomer is strongly quenched by the dimer, we conclude that:

- The long-lived component with the lifetime $c a$. 3.7 ns which corresponds to that observed in the neat solvent, can therefore, be attributed to the lifetime of $\mathrm{Fl}$ in the solvent-rich regions in the solution.

- The short-lived component with the lifetime $c a .0 .6$ ns can thus be attributed to the fluorescence lifetime of $\mathrm{Fl}$ in the polymer-rich regions where the Fl dimers might be formed due to the low solubility of the probe in the polymer-rich region.

As shown in Figure 6, this two-state model is also supported by the change in the two fractions of the long 


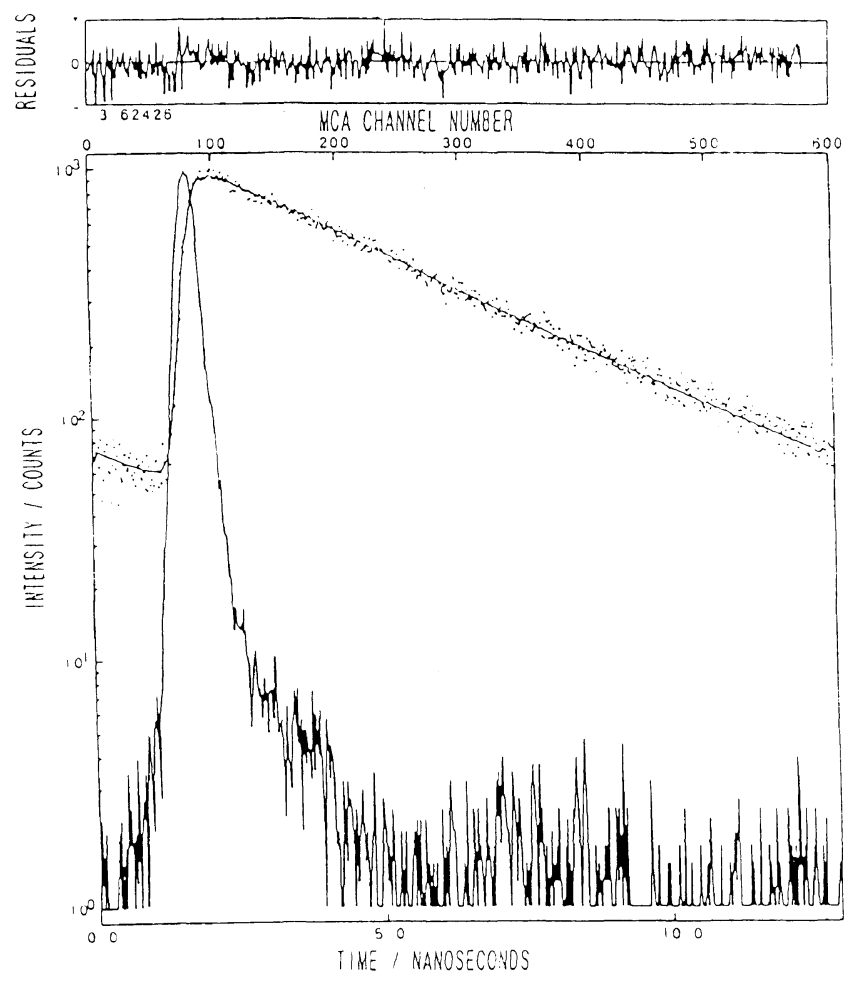

(a)

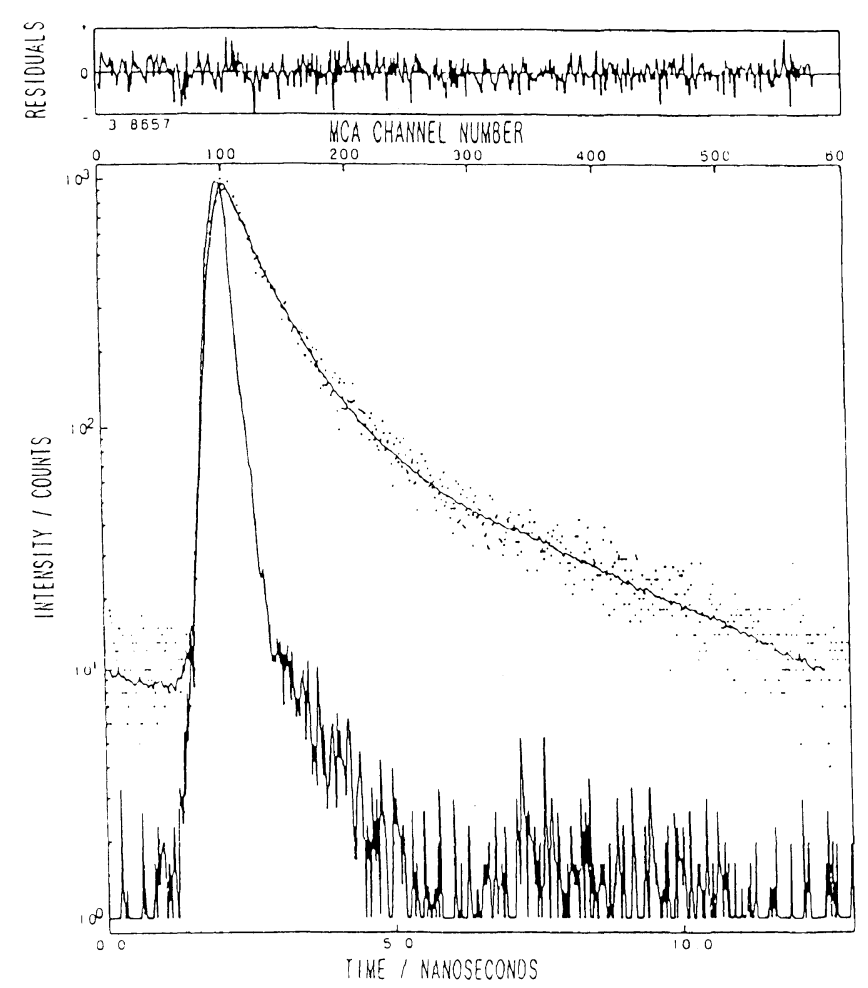

(b)

and short-lived components of the fluorescence decay upon increasing the polymer concentration. As the SPUU concentration increases, the fraction $F_{2}$ of the longlived component which corresponds to the solvent-rich regions, decreases whereas the fraction $F_{1}$ of the short-lived component corresponding to the polymerrich regions increases. These results provide further evidence for the existence of the two micro-environments

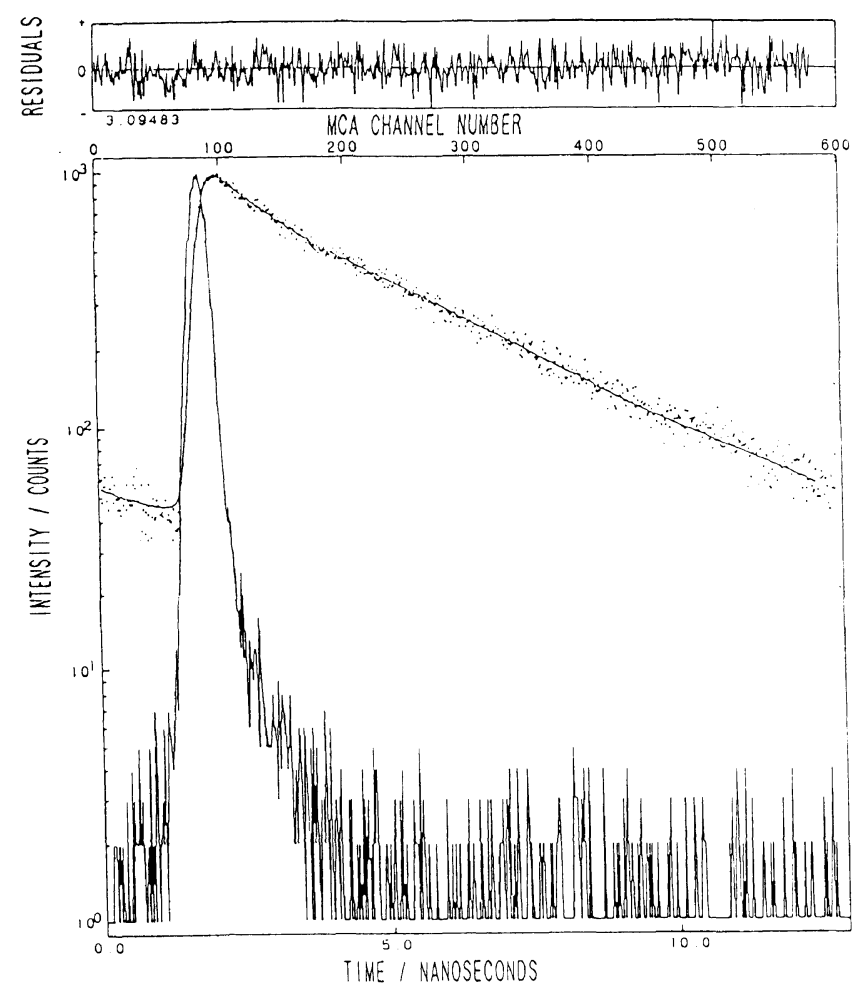

(c)

Figure 5. Fluorescence decay curves obtained for $\mathrm{Fl}$ at $26^{\circ} \mathrm{C}$; (a) in neat solvent; (b) in $20 \mathrm{wt} \%$ polymer solution; (c) in the soft segments (PTMG). For reference, the profiles of the exciting pulse are shown in the same figure $\left(\lambda_{\mathrm{ex}}=488 \mathrm{~nm}, \lambda_{\mathrm{em}}=544 \mathrm{~nm}\right)$.

Table II. Best fit parameters for the fluorescence decay curves shown in Figure 5

$$
I(t)=F_{1} \exp \left(-\frac{t}{\tau_{1}}\right)+F_{2} \exp \left(-\frac{t}{\tau_{2}}\right)
$$

\begin{tabular}{lcccc}
\hline \multicolumn{1}{c}{ Sample } & $F_{1}$ & $F_{2}$ & $\tau_{1}$ & $\tau_{2}$ \\
\hline Neat solvent & & 1.00 & & 3.80 \\
20wt \% solution & 0.58 & 0.42 & 0.66 & 3.50 \\
Soft-segment & 0.06 & 0.94 & 0.61 & 3.60 \\
\hline
\end{tabular}

in the SPUU solutions detected by the excited state of Fl. On the other hand, fluorescence lifetimes $\tau_{1}$ and $\tau_{2}$ do not significantly change with the SPUU concentration within the range $5-30 \mathrm{wt} \%$ as depicted in Figures 7 (a) and $7(\mathrm{~b})$. This dependence of fluorescence lifetime on the polymer concentration is in accordance with the dependence of fluorescence intensity on the SPUU concentration obtained by photostationary excitation as shown in Figure 8.

Rotational Relaxation of Fluorescein in SPUU Solutions and the Aggregation of Polyurethaneurea in DMAc

$\mathrm{Fl}$ can be considered as an isotropic rotor, i.e., a pseudo-spherical molecule, with its absorption dipole parallel to the emission dipole. As a result, the rotational diffusion of $\mathrm{Fl}$ is isotropic and can be expressed by a single relaxation time $\rho$ which is related to the anisotropy ratio $r$ by the Perrin equation ${ }^{12,13}$. 


$$
\frac{r_{0}}{r}=1+\frac{3 \tau}{\rho}
$$

where $r$ is the anisotropy ratio which was experimentally obtained from steady-state measurements. $r_{0}$ is the limiting anisotropy which is taken as 0.4 for the case of fluorescein. ${ }^{14} \tau$ and $\rho$ are respectively Fl lifetime and the rotational relaxation time of Fl. Equation 3 is obtained by numerical integration of the reorientational correlation function obtained from a three-dimensional rotational diffusion equation over time ranging from zero to infinity. Since the fluorescence lifetimes of the Fl are almost unchanged within the range of SPUU concentrations used in this study, the dependence of the steady state anisotropy ratio $(r)$ can be used to discuss the structural changes accompanying an increase in polymer concentration.

Figure 9 shows the dependence of the steady-state anisotropy ratio of $\mathrm{Fl}$ on the concentration of SPUU

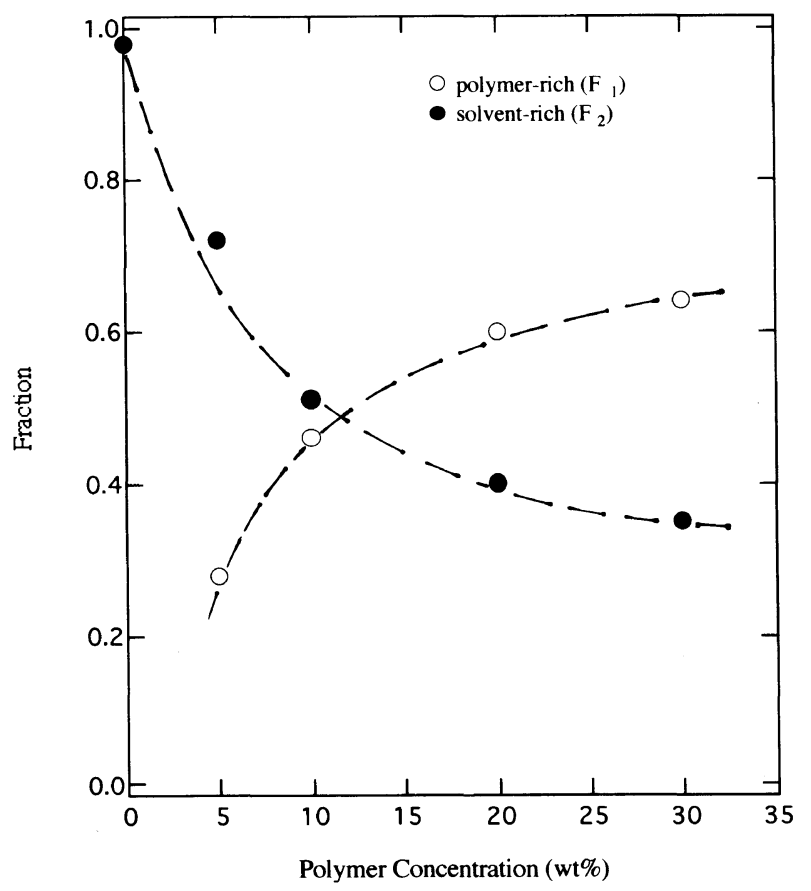

Figure 6. Dependence of the fractional contribution of the 2 components of the decay curve on SPUU concentration.

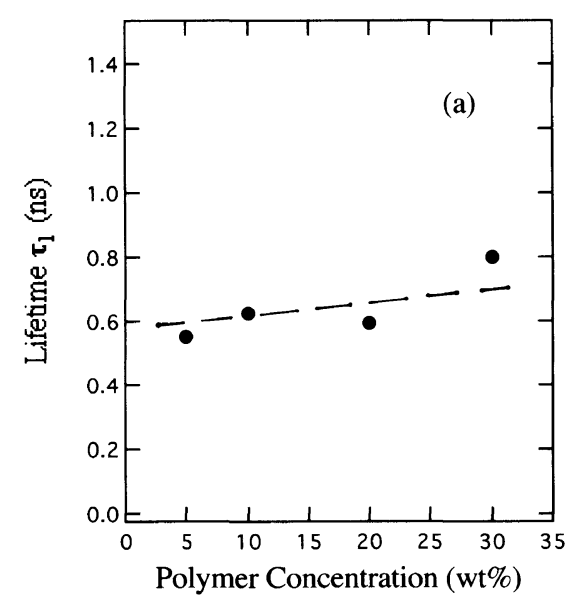

in the range $2-30 \mathrm{wt} \%$ obtained by photostationary excitation. Obviously, there exist transitions in the vicinity of 3 and $15 \mathrm{wt} \%$ of SPUU obtained at $26^{\circ} \mathrm{C}$. The transition in the vicinity of $3 \mathrm{wt} \%$ can be attributed to the onset of the concentration at which the anisotropy of fluorescein becomes sensitive to polymer segments in its vicinity, (below this concentration, the anisotropy ratio of fluorescein in SPUU solutions is equivalent to that in neat solvent). The transition in the vicinity of $15 \mathrm{wt} \%$, on the other hand, would reflect some structural changes in the solutions upon increasing the polymer concentration at the experimental temperature. In order to elucidate this transition in the anisotropy ratio of $\mathrm{Fl}$, SAXS of the SPUU solutions was undertaken under the same experimental conditions. The microphase separation of the solution can be confirmed from the emergence of a significant peak in the Kratky plots obtained for a $25 \mathrm{wt} \%$ SPUU solution as shown in Figure 10 where the product of the scattering intensity $I(q)$ and the square of the magnitude of the scattering vector $q^{2}$ was plotted versus $q$. Below this particular concentration, no any characteristic scattering was observed. Similar critical behavior of SAXS has been reported previously for a number of analog SPUUs in DMAc. ${ }^{4}$ These SAXS results, indicate that the microphase separation in SPUU solutions takes place in the vicinity of $25 \mathrm{wt} \%$ for the SPUU used in this study. This critical concentration is slightly higher than the concentration $15 \mathrm{wt} \%$ observed by fluorescence depolarization of $\mathrm{Fl}$ for the same solutions.

From these experimental results, it can be concluded that fluorescence depolarization of $\mathrm{Fl}$ is sensitive to the onset of microphase separation in SPUU solutions. The transition in the anisotropy ratio shown in Figure 9 reflects the onset of the microphase separation of the SPUU solutions. This critical concentration is somewhat lower than that detected by SAXS. One of the reasons responsible for the difference in the critical concentrations observed by these two methods would be that fluorescence depolarization provides dynamical information within the excited lifetime of the probe whereas small-angle scattering measurements such as SAXS reveal the static information. For the fluorescence lifetime less than $10 \mathrm{~ns}$, the rotational diffusion of $\mathrm{Fl}$ is only sensitive with the structural changes at the order of

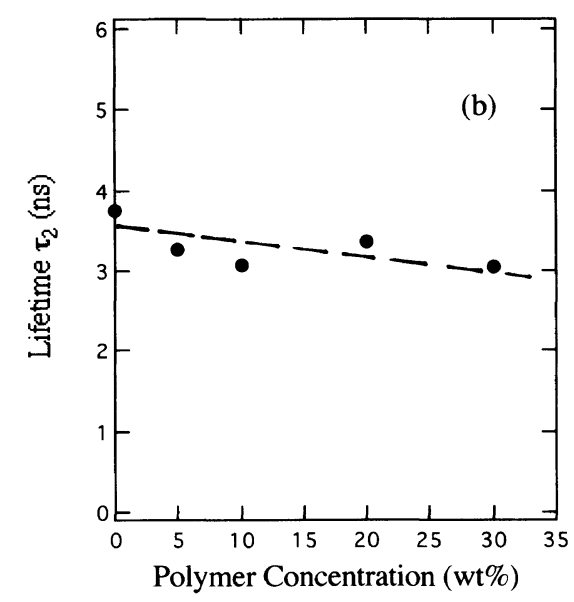

Figure 7. Dependence of the short-lived (a) and long-lived (b) components of $\mathrm{Fl}$ on SPUU concentration observed at $26^{\circ} \mathrm{C}$. 


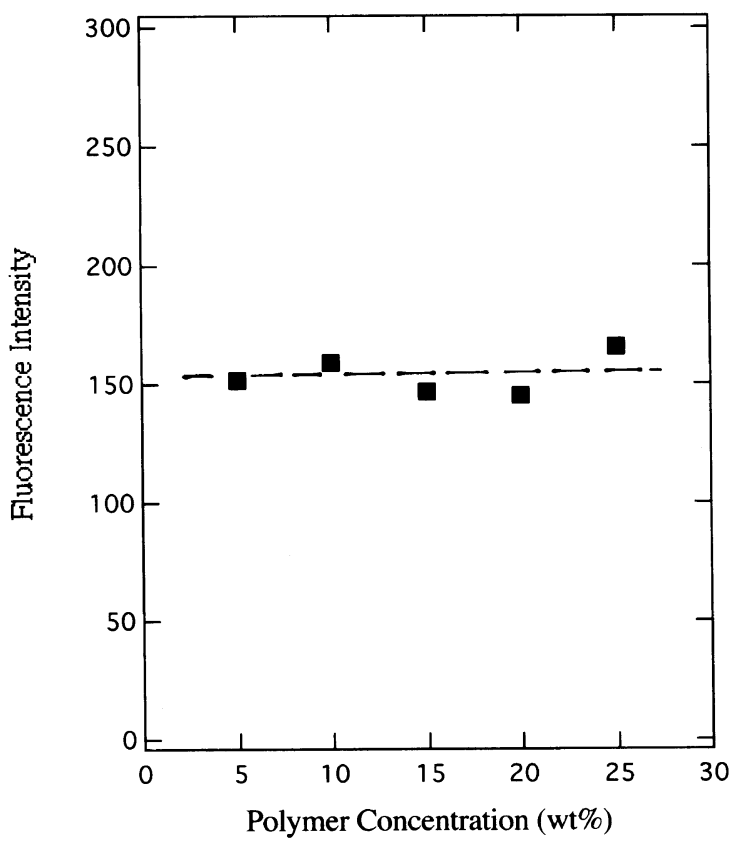

Figure 8. Dependence of the fluorescence intensity of Fl on the SPUU concentration.

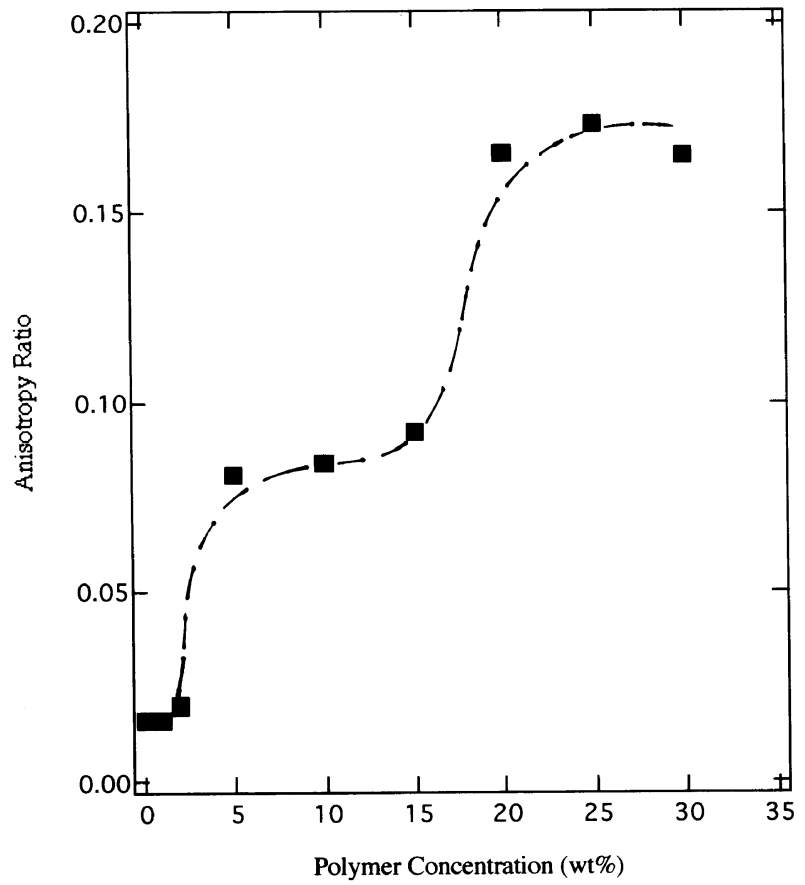

Figure 9. Variation of the fluorescence anisotropy ratio of Fl with SPUU concentration. The data were taken at $26^{\circ} \mathrm{C}$.

$100 \AA$. This scale might be strongly reduced for shorter fluorescence lifetime. Therefore, the onset of microphase separation can be observed earlier by Fl than by SAXS where the characteristic length scale corresponding to the maximum in the Kratky plot of Figure 10 is $c a .190 \AA$ as estimated from the Bragg condition.

\section{SUMMARY}

Microphase separation process of polyurethaneurea in dimethylacetamide was studied by fluorescence depolarization of Fl. The following results were obtained:

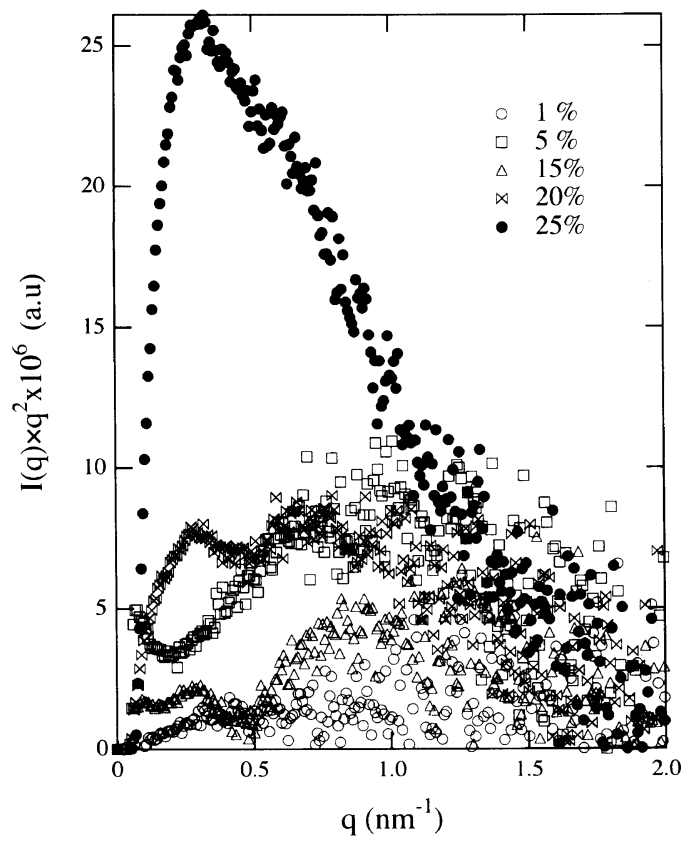

Figure 10. Kratky plots for SPUU solutions with various concentrations observed at $26^{\circ} \mathrm{C}$ by small-angle X-ray scattering.

1) The onset of microphase separation in SPUUs can be detected by fluorescence depolarization using molecular probes such as $\mathrm{Fl}$.

2) The onset detected by fluorescence depolarization emerges at the polymer concentration lower than that observed by SAXS.

The experimental results described above indicate that fluorescence depolarization can be utilized as a useful technique to study the dynamics of structural transition in SPUU solutions. Experiments using fluorescent probes directly labeled to either hard and/or soft segments of SPUU are in progress and will be reported later.

Acknowledgments. The authors would like to thank Professors S. Hirayama and A. Murakami for useful suggestions during the course of this study. We are also indebted to Drs. S. Sakurai and H. Umeda for helping with the SAXS experiments. L.B. is grateful to the scholarship of the Ministry of Education, Science, Sports and Culture of Japan (MONBUSHO) for the Graduate Program of Foreign Students.

\section{REFERENCES}

1. W. Meckel, W. Goyert, and W. Wieder, in "Thermoplastic Elastomers", 2nd ed, G. Holden, N. R. Legge, R. Quirk, and H. E. Schroeder, Ed, Hanser, New York, N.Y., 1996, Chapter 2.

2. S. Abouzahr, G. L. Wilkes, and Z. Ophir, Polymer, 23, 1077 (1982).

3. J. T. Koberstein, A. F. Galambos, and L. M. Leung, Macromolecules, 25, 6195 (1992).

4. M. Shibayama, T. Kawauchi, T. Kotani, S. Nomura, and T. Matsuda, Polym. J., 18, 719 (1986).

5. A. J. Pesce, C.-G. Rosen, and T. L. Pasby, Ed., "Fluorescence Spectroscopy. An introduction for Biology and Medicine," Marcel Dekker, New York, N.Y., 1971.

6. A. Murakami, M. Nakaura, Y. Nakatsuji, S. Nagahara, Q. Tran-Cong, and K. Makino, Nucl. Acids Res., 19, 15, 4097 (1991).

7. T. Minami and S. Hirayama, J. Photochem.Photobiol. A, Chem., 53, 11, (1990). 
8. I. L. Arbeloa, J. Chem. Soc., Faraday Trans. 2, 77, 1725 (1981).

9. K. K. Rohatgi and G. S. Singhal, J. Phys. Chem., 67, 2844 (1963).

10. P. Murkerjee and A. K. Ghosh, J. Phys. Chem., 67, 193 (1963).

11. R. F. Chen and J. R. Knutson, Anal. Biochem., 172, 61 (1988).

12. M. R. Eftink, in "Fluorescence Spectroscopy. 2. Principles," J.
R. Lackowicz, Ed., Plenum Press, New York, N.Y., 1991, Chapter 2.

13. Y. Nishijima, J. Macromol. Sci. Phys., B8, 389 (1973).

14. M. U. Kumke, Guang Li, and L. B. McGown, Anal.Chem., 67, 3945 (1995). 